ISSN 1816-6075 (Print), 1818-0523 (Online)

Journal of System and Management Sciences

Vol. 11 (2021) No. 4, pp. 127-145

DOI:10.33168/JSMS.2021.0407

\title{
A Study on Blockchain Interoperability Mechanism
}

\author{
Qing Zhou ${ }^{1}$, Youngseok Lee ${ }^{2, *}$ \\ ${ }^{1}$ Jiujiang University,551 Qianjin East Road, Lushan District, \\ Jiujiang City, Jiangxi Province, China \\ ${ }^{2}$ Department of Information \& Telecommunication Engineering, Kunsan National \\ University, 558 Daehakro, Kunsan City, Jeonlabukdo, South Korea \\ ${ }^{1}$ zhouqing1418@gmail.com; ${ }^{2}$ leeys@kunsan.ac.kr
}

\begin{abstract}
Over the past decade, blockchain has developed quickly and widely accepted as a value transfer infrastructure. Today, there are more than 60,000 blockchain projects that cover almost all aspects of our daily life. However, these distributed application logics are isolated from each other, and neither impossible to integrate into one single blockchain. With the increasing popularity of blockchain applications, there is a growing tendency to become a new silo of value, and the need for interoperability between blockchains is rapidly emerging. Since blockchain was originally designed to be a self-sufficient and complete system, and the decision-making depends on group consensus rather than a single entity, the data consistency and scalability issue has become the bottleneck that hinders the development of blockchain technology. In recent years, the concept of interoperability has been investigating quite intensively, and there are also a few cross-chain projects that come into the field of vision. Currently, there is no standard solution for cross-chain interoperation, and also little theoretical progress on this topic. In this paper, we propose a general cross-chain solution based on the idea of modularity, abstraction, and layering, which decoupling the cross-chain function from the consensus algorithm and specific application logic, and utilize a Merkle proof to ensure the validity and legality of cross-chain operations. Since the underlying implementations of homogeneous and heterogeneous blockchains are different, we treat them separately. For homogeneous blockchains, we suggest a TCP-like cross-chain transport protocol (CCTP). While for heterogeneous blockchains, we present a method to construct the relay chain to realize the crosschain function. The proposed scheme can enable the correct, effective, reliable, orderly, and timely transmission of cross-chain data. However, the essential difference between the operations within a single blockchain and the interoperability between different blockchains is that the trust domain is different. Cross-chain interoperation itself breaks the completeness of the blockchain, therefore, some efficiency and safety must sacrifice to trade-off.
\end{abstract}


Keywords: Middle-income group, housing affordability, multi-ownership housing, Sonadanga residential area.

\section{Introduction}

Blockchain technology as a trusted machine participating in the credible society construction has been universally accepted after more than ten years of evolution and development. With the increasing maturity of blockchain technology, the application scenario is increasing exponentially. However, the reality of society consists of many different industries and economic fields. It is not realistic to build all the business in diverse areas on one single blockchain, which is more practical for each industry or field to establish its economic circulation systems separately. While blockchain has brought us great convenience, it has also encountered some obstacles. Of all the barriers to the blockchain, cross-chain challenge constrains the development space to the greatest extent. So far, there is no universal solution for the exchange of assets and arbitrary data transfer between different blockchains, which is because cross-chain operations are complex to implement due to the closed nature of blockchains. Ultimately each blockchain naturally evolves into a separate island of value or information. The structure of different chains is highly heterogeneous, and data and assets are not interchangeable. For both public and private block chains, the implementation of cross-chain technology is the most critical factor to facilitate the transfer of value between chains. The technology is dedicated to freeing blockchain from a single value island and building bridges that communicate with the outside world and expand outwards. Value cannot transfer between two blockchains but between two specific users or accounts. If consensus mechanism is the soul of blockchain technology, then cross-chain technology is the key to realize value transfer.

Up to date, there has been very little theoretical progress in interoperability, while the requirements for interoperability and efficiency are increasing as blockchain systems evolve. Cross-chain value exchange and data transmission have some particular demand for security. Cross-chain interoperability itself breaks the trust domain of blockchain, and the group consensus decision mechanism also has the risk of a fork. Therefore, the data transmitted across chains should be accurate, timely, orderly, fast, effective, anti-rollback, and so on.

Based on the current mainstream blockchain platform and existing cross-chain technology research, in this paper, the requirement details to achieve cross-chain interoperability are further analyzed and summarized, and then puts forward a common cross-chain scheme for isomorphic blockchain and heterogeneous blockchain respectively. Based on this scheme, developers can implement the crosschain logic, and users can build their cross-chain modules and define a series of interoperation protocols. We can also perform cross-chain interoperation as simple as a local function call. Although this scheme can realize the accurate, orderly, fast, and verifiable transmission of cross-chain data from the source chain to target chain, 
the implementation process is not easy. There are some limitations in security and efficiency. The cross-chain efficiency and difficulty of realization depend heavily on the degree of single-chain standardization. So far, there are no related successful use cases put into use, and either there are no uniform standards, related theoretical research is lacking. With blockchain bottleneck technology, the topic deserves more in-depth exploration.

\section{Related Works}

Since the advent of blockchain technology, represented by Bitcoin, in 2008, it has grown considerably. Up to date, there are more than 60,000 blockchain projects all over the world. With the blockchains built on different consensus algorithms, data structures, security algorithms, and ledger types, different blockchains were unable to interoperate with each other. Blockchain interoperability technology aims to connect these data silos and deliver trusted values across blockchains. Over the past few years, there have been some innovations in blockchain interoperability and scalability research area, and several studies have been done on this topic.

In the early stages of cross-chain technology development, asset transfer represented by Interledger Protocol (Thomas and Schwartz, 2015) and BTC Relay (Consensys, 2016) received the most attention. Recently, cross-chain infrastructures have occupied most of the stage, of which PolkaDot and Cosmos are the most influential two projects.

In 2008, Nakamoto published a report named Bitcoin: a peer-to-peer electronic cash system, which is considered the origin of blockchain technology, and in a long period since then, blockchain has developed considerably base on one single-chain. However, these variants of different patterns and types have many problems, such as block time and block size, which cannot solve with smart contracts.

In 2012, the Ripple Lab proposed an InterLedger cross-ledger interoperability protocol (ILP) for the first time to address interaction and collaboration between different blockchains through a third-party notary mechanism, adopting a connector based on the Byzantine algorithm to ensure that a payment event occurs correctly. Strictly speaking, it is not only a scalability solution, but it provides an ad hoc interoperation between different ledger systems through a loosely coupled bilateral relationship network. The purpose of ILP is to facilitate payments, especially payments across disparate ledger types. The ILP extends the atomic transaction mechanism to include not only hash locks but also a quorum of notaries.

In May 2013, Herlihy proposed a concept of atomic swap on the BitcoinTalk forum (Herlihy), whereby sub-transactions occur either simultaneously or not, without a third state. This technology has evolved into a popular cross-chain technology called hash locking.

In October 2014, Blockstream put forward the concept of a sidechain for the first time, which uses a two-way Peg sidechain mechanism to realize the transfer of 
crypto assets between the main chain and side chain following an exchange rate (Back. Sidechains allow bitcoins to effectively move from the Bitcoin blockchain to the sidechain and back and allow new features to test on the side chain. The sidechain is the first cross-chain technology that has a giant impact.

In 2015, Bitcoin proposed a Lightning Network (Poon and Dryja, 2016) that uses hash time locking technology to increase its transaction rate through off-chain micropayment channels. The Hash Time Locking mechanism implements a fast transaction channel under Bitcoin et al., 2014)

In 2016, the BTC Relay scheme (Consensys, 2016) was released, and one-way cross-chain communication from Bitcoin to Ethereum was implemented based on a relay chain scheme. In the same year, Vitalik Buterin published an article titled Chain Interoperability, which made a comprehensive and in-depth analysis of blockchain interoperability issue (Buterin, 2016).

In 2017, PolkaDot and Cosmos first proposed to build a blockchain cross-chain infrastructure platform that supports compatibility with all blockchain applications. Fig.1 lists the historical events of the development of blockchain interoperability technology.

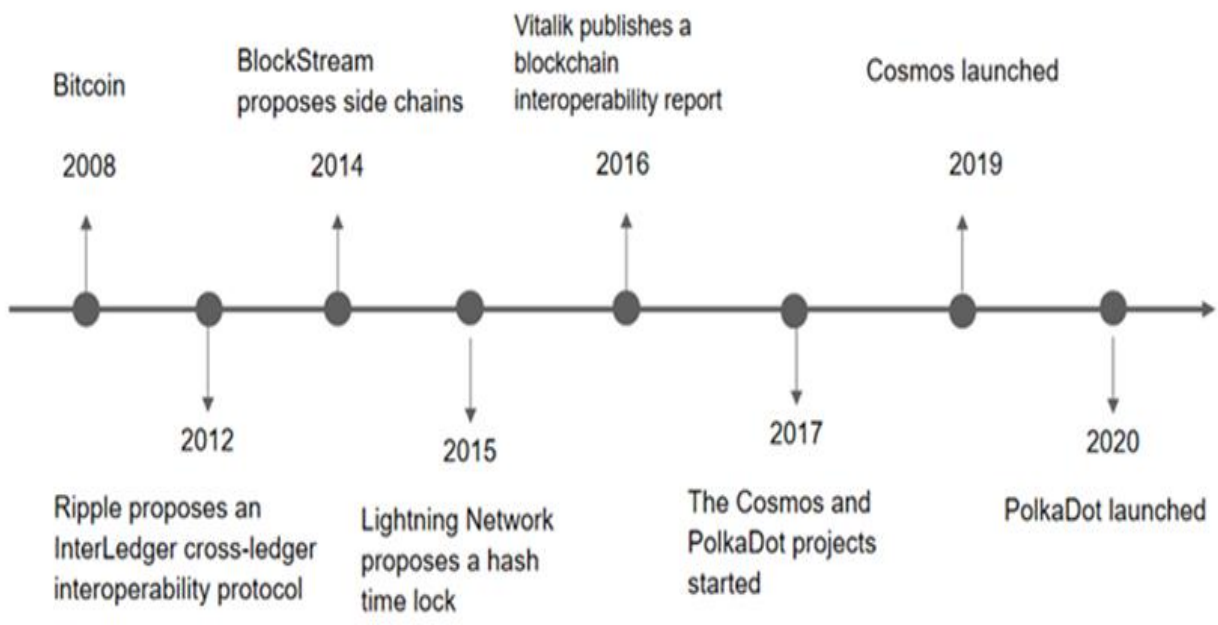

Fig. 1: A timeline of the development of blockchain cross-chain technolog

In 2021, Cosmos finally activated the Inter-blockchain communication protocol that fosters interoperability between Cosmos and other blockchains, while solving scalability issues via shard mechanism.

Today, the Cross-chain Messaging Passing Protocol of PolkaDot is still under construction, which will truly enable arbitrary value transfer between different blockchains.

Among the above cross-chain solutions that already exist, cross-chain 
infrastructure based on sidechain/relay chain is the most promising for large-scale commercial applications, for the scope of application much broader, and supports isomorphism or heterogeneous chain connectivity.

Existing cross-chain technologies have some flaws in terms of security and efficiency. The technical principle and implementation mechanism of cross-chain have their security defects, and there are security problems such as trust dependence and malicious transactions in design and implementation. The structure and characteristics of blockchain systems also have a significant impact on cross-chain security. Nowadays, many countries have developed token exchange and crossborder payment technology in the financial field. However, the further realization of heterogeneous blockchain communication needs more research.

\section{Theoretical background}

Blockchain uses two important hash data structures, the hash list and the Merkle tree, to ensure that data cannot be tampered. In this section, we will briefly describe the two data structures and their common usage. These two data structures are also critical when designing a cross-chain interoperability solution.

\subsection{Hash list}

Hash list is a list of blocks of data that are hashed. It is an extension of a single item hash, often used in file storage. Splitting a file into multiple parts, calculating the hash code for each part, and then storing all of them in one list constitutes a hash list. Hash lists are used for many different purposes, including fast table lookup and data integrity verification.

In blockchain area, all blocks are linked together in chronological order to form a complete chain, called a hash list. Blocks can gradually attach to the one-way chain, and when a new block generates, it will append after the last block, and the one-way link can also backtrack all transaction information that occurred.

\subsection{Merkle Tree}

Merkle tree (MT) is a hash binary tree invented by Ralph Merkle in 1979 (Goes). It is a method of organizing data that allows rapid sorting and validation of large amounts of data. Merkle tree is primarily used to compare and validate data in distributed environments. Such tree data structures can significantly reduce data transmission and computational complexity. Merkle tree is also the most important data structure in blockchain area, and it is adopted to organize transactions in each block. It is the core component of the block, accounting for more than $96 \%$ of the block storage, and the most typical application is the light client that simplifies the payment verification problem.

In Merkle Tree, each leaf node is the hash of a data block, and each non-leaf node is a hash of its sub-node hash. The process of building a Merkle Tree is to hash the combination of the hash values of the left and right child nodes from the lowest data 
blocks climbing up to the top until there is only one node left in the current layer. In general, each node in Merkle Tree has two sub-nodes. If there is a special specification, Merkle Tree can be a multi-fork tree, such as the Ethereum Merkle Patricia Trie. Hash trees are a generalization of hash lists and hash chains, and the hash list is the subtree of the Merkle tree.

The Merkel tree has a characteristic that any change in the underlying data will be passed to its parent node and ultimately affect the root. We can think of the root hash as a tree validator: any trifle change in node data in the tree will result in a change in the root data. Hash trees enable efficient and secure validation of the contents of large data structures. Merkle Tree validates data by validating hash values rather than all of the data, and can substantially improve the validation efficiency, therefore widely used in the distributed system data validation area. Merkle tree is the cornerstone of distributed system data validation.

In the Merkle tree, each transaction can be deleted separately, keeping only the hash value of the transaction, which does not change the cryptographic security and integrity of the entire block but can significantly reduce the amount of data. Therefore, there is no need to worry too much about the growth of data in the blockchain.

The Merkle tree is created by getting two nodes from each layer and hashing them to create a parent node. On the one hand, the Merkle tree is calculated from the bottom up, so as long as the hash value of another adjacent node is known, you can calculate up to the root node. On the other hand, the hash value of the root node can be accurately used as the unique summary of a group of transactions.

The process for data validation is as follows:

After building a series of blocks of data in a distributed system, we can perform top-down data validation by following these steps:

(1) A sends B a hash value that needs to correspond to the root node of Merkle Tree.

(2) B receives this value and compares it to the root hash of the Merkle tree it is building.

(3) If the two values are the same, they represent the same stored content.

(4) Otherwise, B needs to ask A for a hash of the two-child nodes corresponding to the root node.

(5) A sends the corresponding value to B.

(6) B Repeat steps 4 and 5 compared to the hash values in the corresponding node until $\mathrm{B}$ finds one or more data blocks that cause the hash values to be different.

\subsection{Light client}

In the context of blockchain, a client is a piece of software that connects to other clients in a point-to-point manner, which will communicate in a network, and each client is a node, so in some contexts, the client also uses nodes 
instead. There are two types of nodes: full node and light node, of which full node is responsible for verifying and relaying transactions and blocks on the network. However, downloading and verifying the blocks of the entire chain consumes time and resources. On some devices such as mobile phones with limited resources, a light client adopts to indirectly interact with the blockchain network by connecting full nodes. The light client does not need to run all the time, nor does it need to read and write a lot of information to the blockchain. The light node interacts with the full node with minimal trust and is primarily used to validate payments. The light node's block header contains information about the Merkle Tree Root, which is like a digital fingerprint of the account balance in the blockchain and all this information stored in the smart contract. The fingerprint changes when any part of the information changes. Assuming that the majority of miners are honest, the block headers and the "fingerprints" they contain can be considered valid. The light client requests some information from a full node, such as the balance of a specific account. Since the light clients know the "fingerprints" of all blocks, they can verify that the answers given by the full nodes match the "fingerprints" they have. It is a powerful tool that can be used to demonstrate the validity of information without prior knowledge. Compared with intra-chain transactions, cross-chain transactions only account for a few, and there is no need to run a full node, and cross-chain transactions verify through the light client can reduce resource occupation.

\subsection{Merkle proof}

The hash of all the transactions in each block was organized in a Merkle Treelike structure whose Merkle root stored in the block header. The Merkle tree is used to summarize all transactions in each block. Each leaf node is the hash of transaction information, continuously merging the two adjacent hashes into a string and then hashing until only one node at the top, the Merkle root, is deposited in the block header. If there is only an odd number of transactions that needs to be summarized, the final transaction will be copied to form an even number of leaf nodes, thus, a balanced binary tree is constructed. With the Merkle tree, any branch can validate some data effectively.

A Merkle proof is a subsection of a Merkle tree used to prove a given piece of data is a part of a Merkle tree. The process of Merkle proof is constantly hashing together the corresponding hash of the hash and climbing the tree until obtain the root hash, and then compared to the known root hash. Merkle proofs are usually used to decide upon the following three factors:

(1) Whether an input value exists in a Merkle tree;

(2) Prove that a data is part of a dataset without storing all the data in the dataset;

(3) Prove the validity of the data sets contained in large data sets without disclosing the complete data set or its subset.

Similarly, we can concisely prove the membership of a particular block of data in a tree with a hash root. For example, if you want to prove that an MHT tree with a 
hash h contains data, they only need to provide a validator for the block and a series of internal nodes to recalculate the root and compare it to the root $\mathrm{h}$ provided.

\subsection{Simple Payment Verification}

The nodes in the blockchain category consist of full nodes and light nodes. The light node only stores the block header or part of the transaction data, while the full node stores all the block header and transaction data. Some devices like mobile phones cannot store all the data due to the limited storage capacity, and light clients are required to verify the payment on a light node.

During the process of verifying payments, the light node needs to request the hash sequence from the hash of the transaction itself along the Merkle tree to the Merkle tree hash root stored in the block header, through an RPC message to the adjacent full node to confirm the existence and correctness of this transaction. Confirming a transaction in a block of $\mathrm{N}$ transactions requires only a hash value of $\log 2(\mathrm{~N})$ bytes (Merkle, 1987).

Simple Payment Verification refers to payment verification rather than transaction verification, where the two terms are different. Payment verification only needs to verify whether the transaction is confirmed, where transaction verification needs to check whether the transaction meets certain conditions, such as whether the balance is sufficient, whether there is double-spending, and so on. Only if these conditions were met can the transaction pass verification. Transaction verification is much more complex than payment verification, and it is typically done on a full node, while payment validation can carry out on a light client. The goal of SPV is to verify that payment is authentic and how many confirmations it can obtain.

The detailed verification process is as follows:

(1) Calculate the transaction hash value that needs to be verified for payment.

(2) The node acquires and stores all blocks of the longest chain from the blockchain network to local.

(3) The node obtains the Merkle tree hash verification path for the payment to be verified from the blockchain.

(4) Based on the verification path, the root hash value of the Merkle tree be calculated and the result is compared to the root hash value of the Merkle tree in the block in the region.

(5) If consistent, the payment is authentic and valid.

(6) Check the confirmation number received for the transaction according to the location of the block header.

(7) In the blockchain, each block has a Merkle tree, and the leaf node stores the hash value of transactions in the blocks, and each layer recursive can get the Merkle root values. The Merkle root value is stored in the block header to summarize and quickly verify all transaction data in the block. 
The blockchain trilemma. The trilemma of blockchain refers to a public blockchain system that is impossible to satisfy decentralization, safety, and high performance of the three requirements at the same time Fig. 2. Blockchain must make an optimized tradeoff between the three dimensions.

Decentralization refers to the decentralized configuration of a blockchain network, and it is often associated with geographic location, connection patterns, and synchronization of network nodes.

Security refers to the consensus security and anticensorship capability of the blockchain in the presence of malicious nodes. The two concepts correspond to safety and liveness in the conventional distribution consensus.

Scalability refers to multiple aspects: blockchain systems need to maintain security and efficiency as transaction throughput increases and network size grows.

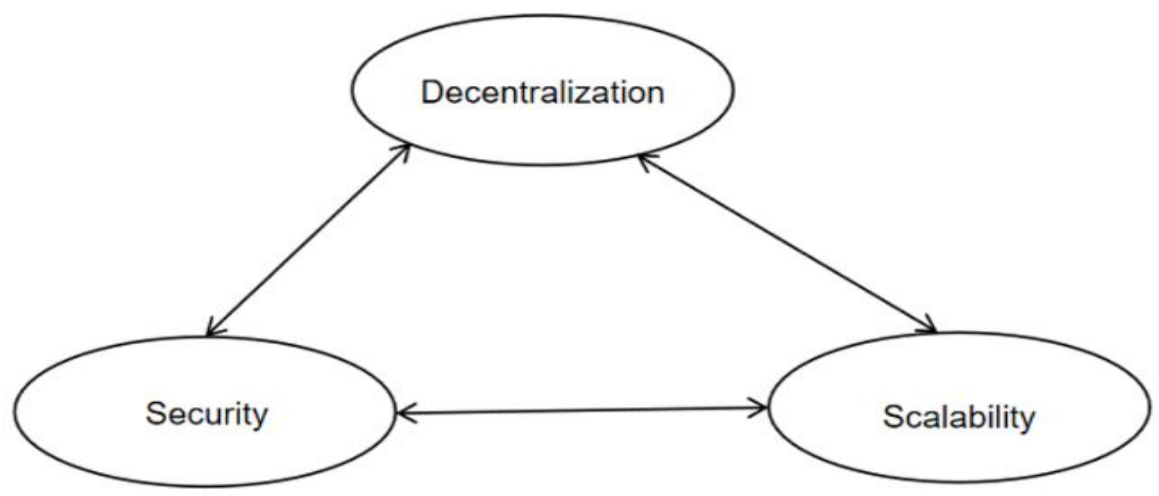

Fig. 2: The blockchain trilemma

Scalability is considered a broader concept of system performance. The essence of cross-chain is blockchain expansion, and common cross-chain scenarios include asset portability, atomic swap, cross-chain oracles, asset encumbrance, and crosschain contracts among others. According to Vitalik Buterin's classification (Poon and Dryja, 2016), there are five types of cross-chain mode, notary schemes, sidechain/relay chain, hash locking, distributed private key control, and other hybrid modes. Of all these schemes, the relay chain solution is the most promising and more likely to be widely replicated.

In recent years, many research efforts have focused on achieving a graceful tradeoff on the trilemma. These efforts can be classified into three directions: the first category is based on consensus mechanisms, the second category is based on block structures, and the third is system-based. The importance of trilemmas for blockchain is that if you talk about one of the other two in the triangle in complete isolation, it is not blockchain at all.

At present, the design of the blockchain was constrained by the trilemma. If cross-chain technology wants to put into large-scale commercial applications, we must break through the trilemma and realize cross-chain utilizing shared and multi- 
chain schemes.

\section{Blockchain interoperability solution}

Cross-chain interoperability refers to the transmission of information, data, and assets between two relatively independent blockchains. As a matter of fact, we have no approach to transfer assets from one chain to another, assuming that the cross-chain transaction initiation chain is A, the destination chain is B. In fact, these assets on blockchain A will not be sent directly to chain B. The essence of cross-chain is to lock a certain number of assets on chain A and then issue an equivalent asset replacement on chain B, and when redemption call smart contract was deployed to destroy this alternative asset, the source blockchain A release previously locked assets, then the difficulty of realizing cross-chain lies on the interoperability of cross-chain messages.

\subsection{Basic requirements to achieve blockchain interoperability}

From the concept and requirements to understand interoperability, the essence of cross-chain is to transfer the message $M$ on chain A safely and reliably to chain B, meanwhile reach the expected effect on chain B. A successful crosschain scheme should solve the following issues:

(1) Authenticity of message $M$, that is, the message $M$ indeed exists on blockchain A, and it does come from chain A.

(2) Routing of message $M$, ensuring that cross-chain messages reach the target chain quickly, accurately, safely, and in an orderly manner.

(3) Proof of the validity of message $M$, where validity refers to whether message $M$ is still valid when it reaches the $B$ chain, which covers mainly three aspects: whether the transferred assets where locked on blockchain A, no double-spending exist, and the state does not change during the data transmission period, etc.

(4) Execution receipt of message $M$. The source blockchain needs to confirm whether the cross-chain interoperability is successful after executing the cross-chain transaction. A confirmation message should return from the source blockchain to the sending blockchain.

(5) Cross-chain transactions did occur on the target chain, and they occured only once.

To address these critical issues, we aim to establish a standard cross-chain solution, just like the TCP/IP protocol for the Internet. The initiative blockchain and response blockchain of cross-chain transactions should at least support these functions as follows:

(1) Both the source chain and destination chain have a queue pair, whose output message queue will be used to handle outgoing messages, and the input queue records incoming messages.

(2) The source chain needs to provide proof of authenticity for cross-chain messages. That is to say when sending cross-chain messages, evidence of 
authenticity is also required. This proof requires building a Merkle tree for crosschain messages and including Merkle root in cross-chain messages or cross-chain blocks. The validation process is similar to simple payment verification. The receive chain rebuilds the Merkle tree after receiving this message, then compares the two Merkle roots to check whether they are the same. It indicates that this message is authentic. Otherwise, it is a fraud message.

(3) A valid route is necessary for the cross-chain message. The routing process needs to build a unified cross-chain information format, specifying the source and destination blockchains of transactions and the contents of messages. The routing information records on the third-party relay chain, and when the receiving chain validates the cross-chain message, they can look it up on the relay chain. The relay chain achieves indirect synchronization of the source chain and the target chain.

(4) Proving the validity of cross-chain information is critical. Cross-chain is not only the transmission of information, and the more meaningful part is the settlement system. In addition to cross-chain data, evidence of ledger status should also send to the receiving chain to prevent double-spending, and both data and evidence should process in strict order. Value transfers occur between specific accounts, not entire ledgers. At present, the KV database-based account storage methods cannot be used to provide proof of validity. Therefore, blockchain needs to design a new verifiable storage structure to make SPV-like validation more convenient. We can consider the UTXO model and the relay chain Merkle tree.

(5) Proof of cross-chain execution results, that is, receipt or acknowledgment, similar to validity, requires the support of a new data structure and operating algorithms.

Cross-chain can be classified as homogeneous and heterogeneous cross-chain according to the underlying technology. Homogeneous blockchains have the same block structure and consensus mechanism, so the destination chain can understand the transaction on the source chain, unlike transactions within the single blockchain, cross-chain transactions across different trust domains. To verify the validity of a cross-chain transaction, we need both a light client and an additional binding Merkle path. In addition, the block location of the initiation chain that is fixed by the genesis block and block height together needs to be recorded for possible rollback operations. Cross-chain between the heterogeneous blockchains is different due to the block structure, and the consensus algorithm of the source chain and target chain is different. The light client of the destination blockchain cannot verify the cross-chain transaction. Hence, we consider adding a state transfer parameter. Although the block structure of the source blockchain is not uniform, we can standardize a state transfer data structure and record the state change results on the relay chain. 


\subsection{Cross-chain between homogeneous blockchains}

Because the security mechanisms, consensus algorithms, network topology, block generation logic, and verification methods in homogenous blockchain are consistent, cross-chain interoperation is relatively simple compared with heterogeneous blockchain. We can achieve this through a TCP-like handshake protocol by changing the status of cross-chain messages and transaction proofs to indirectly synchronize the state of the source chain and destination chain.

\begin{tabular}{|c|c|c|}
\hline Application Layer & \multirow{3}{*}{ Moudle 1} & \multirow{2}{*}{ Moudle 2} \\
\hline & & \\
\hline Smart Contract Layer & & \\
\hline Incentive Layer & Moudle 3 & Moudle 4 \\
\hline Consensus Layer & \multicolumn{2}{|c|}{ Consensus Layer } \\
\hline Network Layer & \multicolumn{2}{|c|}{ Network Layer } \\
\hline Data Layer & \multicolumn{2}{|c|}{ Data Layer } \\
\hline
\end{tabular}

Fig. 3: The hierarchical structure of the blockchain

The ledger format in two homogeneous blockchains is the same, and both the initiation blockchain and response blockchain can understand the respective counterparty's transactions. So similar to a single blockchain, we can also use a light client to verify the cross-chain transaction. Although different blockchains have different trust domains, they all support the underlying P2P network, so we can also use light clients to verify cross-chain transactions. We only need to bind it to a connection between the two blockchains.

According to the conventional six hierarchical structure of the blockchain, we can simply abstract blockchain into the application layer and core layer as shown in Fig. 3 , and then establish a cross-chain protocol between these two layers.

The core layer of homogeneous blockchains is the same. If we can build a protocol between the application layer and the core layer as the cross-chain infrastructure, and in this way, the users can construct their business logic on the application layer, there is no need to worry about the underlying consensus algorithm and the implementation of the cross-chain protocol. The upper incentive layer, the smart contract layer, and all the distributed applications can be regarded as an application module, which is similar to the application layer protocol of the Internet. We can allocate each application module a port number, and bind it to the transmission channel, and establish a cross-chain transmission protocol (CCTP) 
similar to TCP to achieve cross-chain interoperability.

The cross-chain functionality can be modularized to several parts, and each item is compared with TCP as shown in Table I.

Table 1: The comparison between CCTP and TCP

\begin{tabular}{|l|l|c|}
\hline CCTP & \multicolumn{1}{|c|}{ Purpose } & TCP \\
\hline Port & $\begin{array}{l}\text { Each application module or process is assigned a unique port through which } \\
\text { the application can call the underlying transport channel to transmit data. }\end{array}$ & Port \\
\hline Light Client & $\begin{array}{l}\text { Light clients used to track the consensus states of the counterparty blockchain, } \\
\text { according to which together with related Merkle proof can verify the validity } \\
\text { of coming cross-chain transactions. }\end{array}$ & None \\
\hline Connection & $\begin{array}{l}\text { Initialize, establish, update and release a connection between two full nodes of } \\
\text { the source blockchain and the target blockchain, each connection associated } \\
\text { with the client of the counterparty blockchain to verify the validity of the } \\
\text { incoming transaction. Here, the connection was between blockchains rather } \\
\text { than between websites, which requires the support of TCP protocol. }\end{array}$ & None \\
\hline Channel & $\begin{array}{l}\text { Initialize, establish, update and release a channel between two connection end } \\
\text { nodes, initialize a channel to request memory space on the two full nodes, one } \\
\text { connection can establish more than one channel, packets are transmitted } \\
\text { orderly over each channel by using a sequence number. }\end{array}$ & Connection \\
\hline
\end{tabular}

\section{Light client:}

The two participants of the cross-chain transaction first initialize a light client to verify the data transferred from the source chain before sending data, indicating that the chain can verify the validity and legitimacy of cross-chain transactions from the source chain.

\section{Connection:}

Before sending data, two blockchains need to establish a logical connection to connect two chains. For simplicity, we can randomly choose two full nodes to connect. Together with the light client, this connection is responsible for guaranteeing the legitimacy of a cross-chain transaction, that is, confirming that a cross-chain transaction did occur on the target chain and that the cross-chain transaction commits only once.

Channel:

Cross-chain transactions transmit over the channel, on which we can use a sequence number to ensure the transactions delivers in order. Each of the channels maintains four queues to manage the two party's outgoing and incoming messages respectively. When initializing, the protocol requests memory space on two connected full nodes, and more than one channel can be established on a single connection.

The connection can establish using three times hand handshake method similar to the TCP protocol, and the release process utilizes four waves. The channel initialization and release process are the same as the connection. 
Suppose an account on Blockchain A intends to send a cross-chain transaction to another account on Blockchain B, the data need to be transferred, and the process are as shown in Fig. 4.

(1) The user sends a cross-chain transaction from the source account to Blockchain A, on which the transaction is executed, and locks the appropriate amount of assets.

(2) Write the cross-chain transaction to A's outgoing message queue, whose functions are like a mailbox where all the cross-chain transactions place.

(3) To notify the destination blockchain of the events that occurred on blockchain A, a relay-chain is required to forward the cross-chain transaction CTX in A's outgoing message queue to B's incoming message queue. The relay-chain discovers the cross-chain transaction in A's outgoing message queue and the corresponding Merkle Proof through polling or listening mechanism, and then packages the transaction and proof information together into a CrosschainTxPacket and forwards to blockchain B. Meanwhile, it queries the block information where CTX locates, packages the blockhead information into a CrosschainBlockheaderPacket, and sends it to the blockchain B.

(4) After receiving CrosschainTxPacket, blockchain B will verify and execute these transactions. First, it verifies whether the block header on the A chain passes through the consensus process by validators. Then, it verifies whether the Merkle proof of the cross-chain transaction in the CrosschainTxPacket is equal to the block header hash in the CrosschainBlockheaderPacket. When all verifications passed, the $\mathrm{B}$ chain starts to perform corresponding operations, such as generating assets on blockchain $\mathrm{B}$, returning transaction receipts, etc.

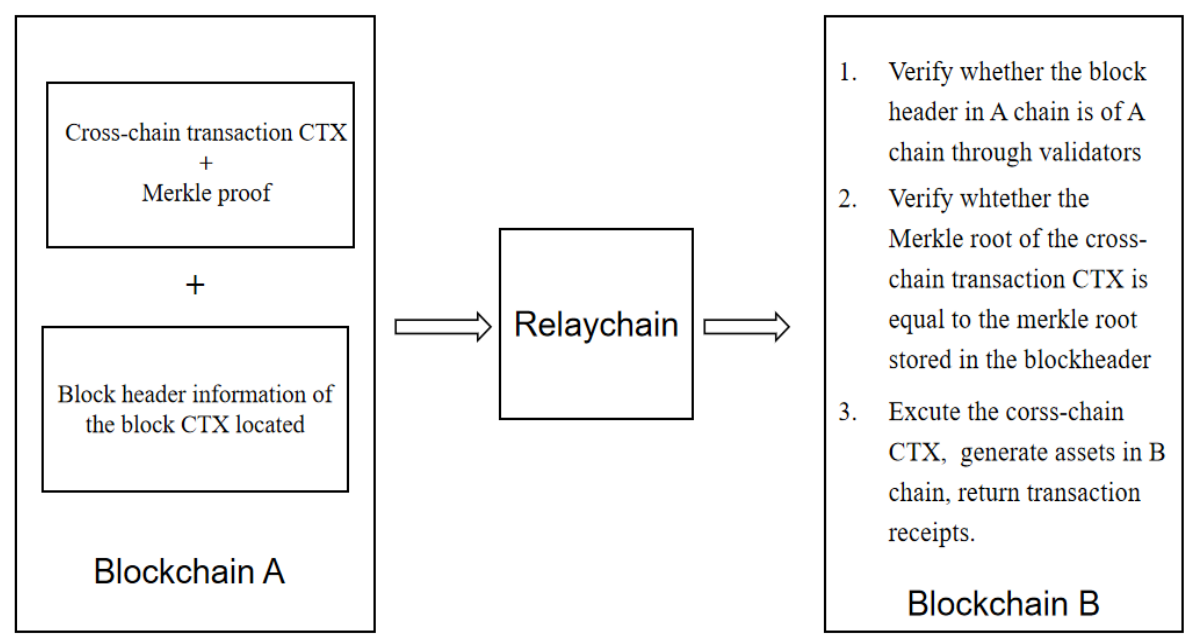

Fig. 4: Cross-chain data for homogeneous blockchain interoperation 


\subsection{Cross-chain between heterogeneous blockchains}

Blockchains based on probability consensus algorithms such as PoW and PoS are significantly different from those based on traditional determinism consensus algorithm BFT in terms that block generation and final confirmation mechanisms. Therefore, it is not easy to design cross-chain protocols between heterogeneous blocks. Generally, a third-party relay chain is needed to assist.

Since there is no blockchain to rely on another blockchain to determine the validity of transactions, interoperability is an inherent contradiction in blockchain, and we have to make some additional assumptions to achieve cross-chain interoperability.

A decentralized blockchain A interoperable with a blockchain B is equivalent to a decentralized blockchain C containing both A and B's ledgers [12]. That is to say, to realize cross-chain interoperation on heterogeneous blockchain equivalence to construct a blockchain covering the source chain and destination chain, this chain is commonly known as a relay chain.

If chain A sends a message $M$ to chain $B$, a channel needs to initialized between block $\mathrm{A}$ and block $\mathrm{B}$ to transmit the cross-chain transaction and the corresponding proof information. In addition to the cross-chain message itself, corresponding proof of validity and state transfer suggestions should also be included. Fig.5 identifies all the data that needs to be transmitted across the source and destination chains.

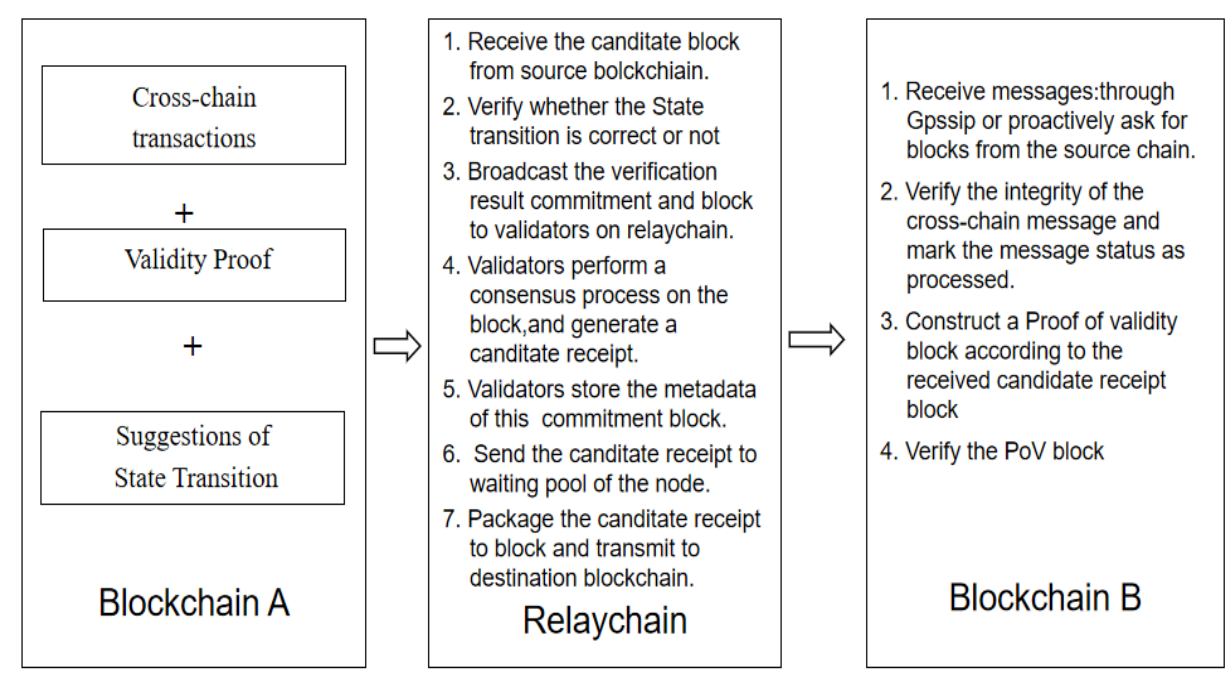

Fig. 5: Cross-chain data for heterogeneous blockchain interoperation

Cross-chain messages are collected by the sending chain and then verified, packaged into blocks, and reach consensus among validators.

When a relay chain validator receives an uncommitted block and proof of validity 
from the source blockchain, it checks that this block follows the state transition rules of the source chain. It only needs to: verify the list of state transitions carried in the candidate block, the values in the source chain database that the block modified, and the hash of the unmodified nodes in the Merkle tree. The validator does not need to check each value in the parallel chain state, only the modified value to ensure that the modification is valid. If passed, it signs and broadcasts proof of validity to other validators assigned to the source chain. Once the validator's consensus verification pass indicates it is a valid delivery, they will construct a "candidate receipt."

To synchronize the status of the initiator chain to the relay chain reliably, each block generated by the initiator chain generates a corresponding proof of validity block. The verifier node on the relay chain verifies this validity block through the algorithm provided by the initiator chain. If the verification passed, the block header and related metadata of the initiator chain was written into the relay chain, which is equivalent to the state of the initiator chain synchronized to the relay chain. The proof of validity block consists of all transactions, data read when processing each transaction (such as transfer sender balance) and the validity proof, and data written when processing each transaction (such as updating transfer sender B balance) and its proof. In this way, the verifier does not need to store any initiator chain state and only needs to re-execute the transaction.

Considering the implementation of validity proof and state transition verification need Merkle proof, we can organize the hash value of cross-chain transactions into a Merkle tree structure, utilizing a message hash chain that tracks the cross-chain messages. All the message hash chains constitute a Merkle tree, and the path from the Merkle root to the leaf node is a channel. We use the message hash list rather than the message chain because the hash chains have a property, which is when we delete a leaf node, the integrity of the data will not change.

The status of the cross-chain messaging channel is maintained by the relay chain using a two-dimensional table whose row represents the source chain, and the column represents the destination chain. Each item of the channel tracks the latest sender message queue root and the relay chain block number. Fig.6 is an overall diagram of cross-chain message access flow.

Under the method to build a blockchain, relay blockchain also needs to construct its block generation algorithm and consensus algorithm.

On the source blockchain end, all the hash values of the cross-chain message construct a Merkle tree. Fig.6 shows the details. The leaf node of the Merkle tree is a triple $(\mathrm{H}(\mathrm{Mi}), \mathrm{b}, \mathrm{H}($ Previous-header) $)$, where $\mathrm{H}(\mathrm{Mi})$ represents the hash value of the cross-chain message $\mathrm{Mi}, \mathrm{b}$ is the block number of the relay chain at which the parent message emit, which can use as the global time, $\mathrm{H}$ (Previous-header) is the hash value of Mi's parent, and the leaf node H represents a block header for the message queue hash chain (MQHC). Each MQHC is a channel for the source chain 
to the destination chain. All the message queue hash chains construct a Merkle tree, and the root hash MR is recorded into a block and then send to the relay chain.

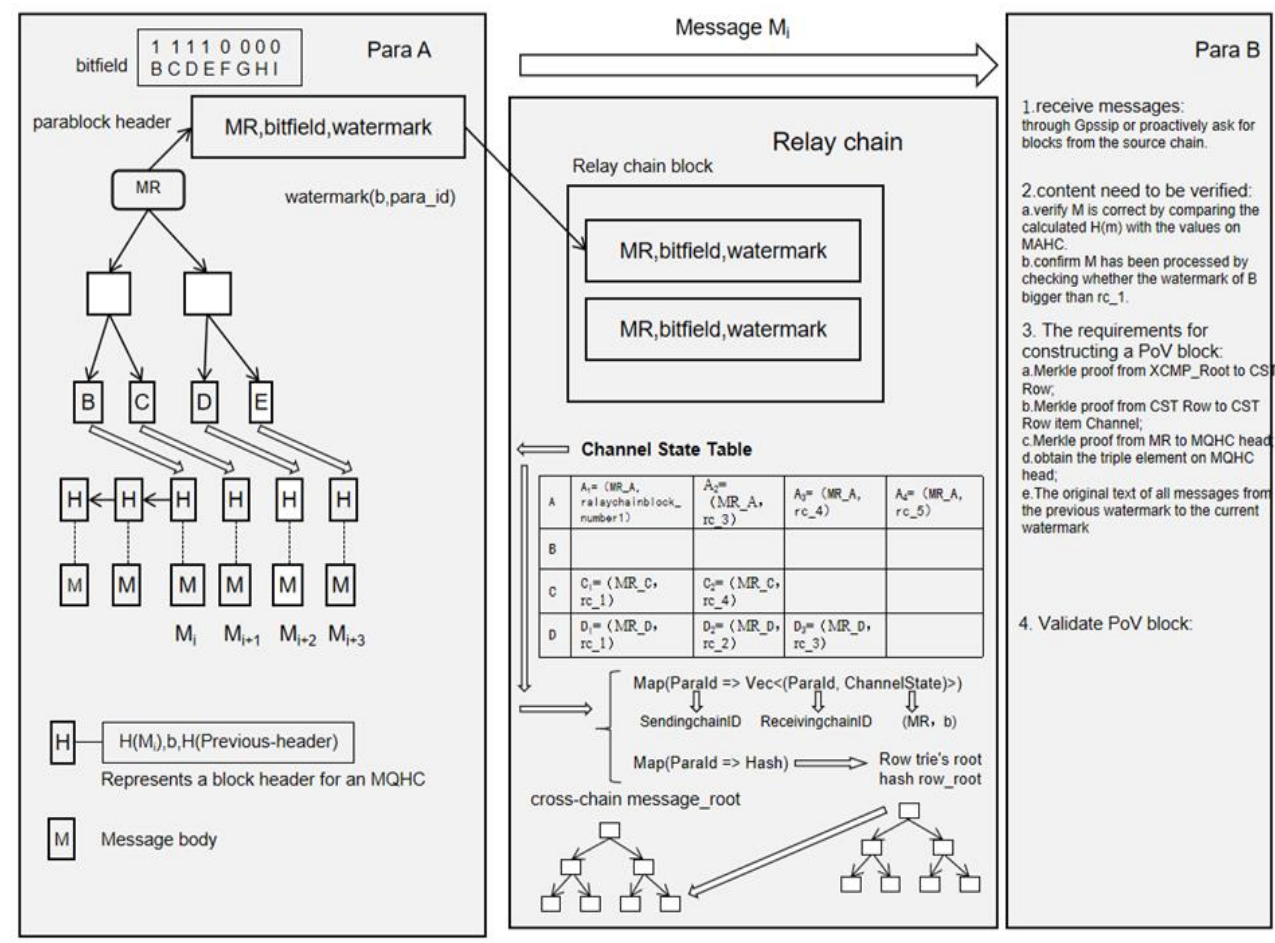

Fig. 6: An overall diagram of cross-chain message access flow

Watermark is used to record the location of the nearest relay chain block processed on the destination chain incoming queue. On the relay chain, there are two important data structures: channel state table (CST) and two maps, each CST item is a tuple (MR, relay chain block number) stored in a row, and each row represents the message that comes from the same source chain. These row items were hashed together to construct a row-root, and whenever a single data item changes in a row, its row-root changes, then further construction of the Merkle tree with these row-roots as leaf nodes can fully reflect the state roots of the relay chain.

On the destination chain, the validator node will reconstruct a block according to the original message in the outgoing message queue, block header of hash message chain, the Merkle proofs on relay chain, and the Merkle proofs on source chain. The validators will also verify this block and commit it, and the block header will update to the relay chain. Then, the block header goes up to the relay chain, and the relay chain updates its status. The sending chain also knows that the message it sent has been processed by querying the relay chain. The relay chain updates the state, and the sending chain queries the relay chain to know that the message it sends has finally been processed. 


\section{Conclusion and future work}

In this article, we propose a solution to the blockchain interoperability problem, with the goal of allowing data and assets to move between two independent blockchains on demand. Since the underlying implementations of homogeneous and heterogeneous blockchains are different, we propose a general solution framework. These two approaches are totally different. The solution for homogeneous blockchain is a cross-chain transmission protocol similar to TCP protocol, responsible for forwarding cross-chain messages only. The approach for heterogeneous blockchain is to use a secondary blockchain to facilitate the delivery and validation of cross-chain messages. The crosschain metadata will be stored on the blockchain after reaching the consensus, and the cross-chain messages themselves will directly transfer from the outgoing message queue of the source chain to the incoming message queue. At present, the development of cross-chain technology is still in the initial stage, and there are no cases of large-scale commercial applications. Among the solutions already launched, none of them is perfect, and each of them has defects. The research in this paper is just a beginning, and more details and implementation methods are worth further exploration.

\section{References}

Back, A., Corallo, M., Dashjr, L., Friedenbach, M., Maxwell, G., Miller, Poelstra, A., Timón, J., \& Wuille, P. (2014). Enabling Blockchain Innovations with Pegged Sidechains, Oct 22 (2014).

Berman, P., Karpinski, M., \& Nekrich, Y. (2007). Optimal trade-off for Merkle tree traversal. Theoretical Computer Science, 372, 26-36.

Buterin, V. (2016). Chain interoperability, R3 Research Paper, (2016). https://www.r3.com/wp-content/uploads/2017/06/chain_interoperability_r3.pdf.

Consensys. (2016). BTC Relay Documentation Release 1.0. https://buildmedia.readthedocs.org/media/pdf/btc-relay/latest/btc-relay.pdf, Sep 10.

Goes, C. I. GmbH. The Inter-blockchain Communication Protocol: An Overview. https://ibcprotocol.org/documentation.

Herlihy, M. Atomic Cross-Chain Swaps.

Lafourcade, P., \& LombardPlatet, M. (2020). About blockchain interoperability. Information Processing Letters, 161, Sep.

Merkle, R. (1987). A Digital Signature Based on a Conventional Encryption Function, CRYPTO. 
Michael, S. (2004). Merkle Tree Traversal in Log Space and Time. International Conference on the Theory and Applications of Cryptographic Techniques. Springer, Berlin, Heidelberg.

Poon, J., \& Dryja, T. (2016). The Bitcoin Lightning Network: Scalable Off-Chain Instant Payments, Jan. 14, (2016).

Thomas, S., \& Schwartz, E. (2015). A Protocol for Interledger Payments, https://interledger.org/interledger.pdf.

Wood, G. Polkadot: Vision for A Heterogeneous Multi-Chain Framework Draft 1, Https://Polkadot.Network/Polkadotpaper.Pdf. 